\title{
Short Note on Computational Intelligent Techniques for Industrial Production Systems
}

\section{P Vasant}

Department of Fundamental and Applied Sciences, Universiti Teknologi Petronas, Tronoh, Malaysia

*Corresponding author: Pandian vasant, Department of Fundamental and Applied Sciences, Universiti Teknologi Petronas, 31750 Tronoh, Malaysia, Tel: 60103819267; E-mail: vasantglobal@gmail.com

Received date: February 11, 2014, Accepted date: February 12, 2014, Published date: February 25, 2014

Copyright: (C) 2014 Vasant P. This is an open-access article distributed under the terms of the Creative Commons Attribution License, which permits unrestricted use, distribution, and reproduction in any medium, provided the original author and source are credited.

\section{Editorial}

Computational intelligent has been accepted and recognized by global research scientists, decision makers and practicing researchers in recent years as powerful artificial intelligent techniques, for solving unlimited number of complex real-world problems particularly related to research area of unpredictable and uncertain optimization. Under the uncertain and turbulence environment, classical and traditional approaches are unable to obtain a complete satisfactory solution with higher level of degree of satisfaction for the real world practical problems on optimization. Therefore, new global computational intelligent optimization methods are required to handle these issues seriously. One such method is hybrid evolutionary computation, a generic, flexible, robust, beneficial and versatile framework for solving complex and unpredictable problems of global optimization and search in real world application problems.

The main significant contributions of this research work are on the construction of a brand new non-linear membership function using fuzzy approach to capture and describe vagueness in the technological coefficients of constraints in the industrial production planning problems [1]. This non-linear membership function is flexible and convenience to the decision makers in their decision making process [2]. Secondly, a nonlinear objective function in the form of cubic function for fuzzy optimization problems is successfully solved by 15 hybrid and non-hybrid optimization techniques from the research areas of computational intelligent and classical approaches [3]. Among the 15 techniques, three best techniques are selected based on the percentage of quality solution respect to level of satisfaction and the degree of possibility. An intelligent performance analysis table is constructed to the convenience of decision makers and implementers to select the niche optimization techniques in order to apply in the real world problem solving approach particularly related to industrial engineering problems [4].
One can conclude that the intelligent hybrid optimization techniques are robust, less time-consuming, dependable, high quality solutions and an efficient productive tool for solving the non-linear real world problem in an unpredictable uncertain environment [5]. The hybrid line search with genetic algorithms and hybrid line search with simulated annealing techniques developed in this research work are user friendly, easy-to-use and can serve as a teaching and research tool, besides being useful for practicing scientist in the research area of industrial engineering [6]. Moreover, the well-developed hybrid optimization techniques in this research work can possibly solve the real world problem of man-made chaotic problems, and natural disasters problems as well as unpredictable uncertain problems in a turbulence environment.

\section{References}

1. Vasant $\mathrm{P}$ (2013) Hybrid optimization techniques for industrial production planning: A review. Handbook of Research on Novel Soft Computing Intelligent Algorithms: Theory and Practical Applications. IGI Global 2: 41-68.

2. Vasant $P$ (2013) Hybrid optimization techniques for industrial production planning. Formal Methods in Manufacturing Systems: Recent Advances. IGI Global 2: 84-111.

3. Vasant P (2013) Hybrid LS-SA-PS methods for solving fuzzy non-linear programming problems, Mathematical and Computer Modelling 57: 180-188.

4. Vasant P (2012) A novel hybrid genetic algorithms and pattern search techniques for industrial production planning. International Journal of Modeling Simulation and Scientific Computing 3: 1-19.

5. Vasant $\mathrm{P}$ (2011) Hybrid mesh adaptive direct search and genetic algorithms techniques for industrial production systems. Archives of Control Sciences 3: 299-312.

6. Vasant P (2013) Hybrid optimization techniques of pattern search and genetic algorithm: A case study in production systems. Int J Hybrid Intell Syst 10: 179-190. 